\title{
Mortalidade de mulheres em idade fértil em um hospital terciário de Recife-PE: um estudo retrospectivo (2015-2019)
}

Mortality of women of fertile age in a tertiary hospital in Recife-PE: a retrospective study (20152019)

Mortalidad de mujeres en edad fértil em um hospital terciario em Recife-PE: estudio retrospectivo (2015-2019)

keiliane Ribeiro de Souza ORCID: https://orcid.org/0000-0002-3235-2399 Universidade Federal de Pernambuco, Brasil E-mail: keiliane.r@hotmail.com

Aracele Tenório de Almeida e Cavalcanti ORCID: https://orcid.org/0000-0001-8134-5438 Universidade Federal de Pernambuco, Brasil E-mail: Aracelecavalcanti@hotmail.com Lais Vasconcelos Santos ORCID: https://orcid.org/0000-0002-9678-4350 Universidade Federal do Rio Grande do Norte, Brasil E-mail: Lais_lvs@hotmail.com

Bruna Joares Aldina Lucena ORCID: https://orcid.org/0000-0003-0870-1031 Universidade Federal de Pernambuco, Brasil E-mail: brunajoares@gmail.com

Nathália Barreto Januário Chaves de Figueiredo ORCID: https://orcid.org/0000-0002-5723-6706 Universidade Federal de Pernambuco, Brasil E-mail: Nathaliabfigueiredo@hotmail.com Yasmin Brisde Aldina Lucena ORCID: https://orcid.org/0000-0002-9617-7921 Universidade Vale do Salgado, Brasil E-mail: yasminbrisdealdinalucena@gmail.com

Alexandre Marlon Carvalho de Oliveira ORCID: https://orcid.org/0000-0002-9278-4329 Hospital Israelita Albert Einstein, Brasil E-mail: Marlonquantica@hotmail.com

\begin{abstract}
Resumo
A mortalidade de mulheres em idade fértil e em período gravídico puerperal correspondem a uma parcela importante de óbitos femininos no país, sendo a mortalidade materna um importante indicador para avaliar a qualidade da assistência à saúde oferecida a população. A hipertensão, hemorragia, infecções puerperais, doenças do aparelho circulatório complicadas pela gravidez, parto e puerpério e o aborto são as principais causas de morte materna no país. Diante disso, o objetivo desse trabalho é descrever os óbitos de mulheres em idade fértil notificados no Hospital Barão

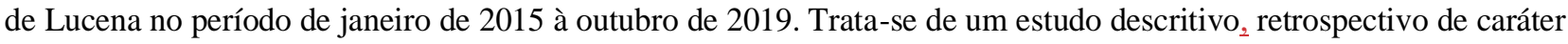
quantitativo, realizado a partir de fichas de notificação de óbitos e prontuários hospitalares de mulheres em idade fértil, de 10 a 49 anos que foram a óbito no Hospital Barão de Lucena. O estudo apresentou um maior número de óbitos de mulheres com idade entre 40-49 anos, da cor parda, baixa escolaridade e sem parceiro fixo. Quanto às causas de morte, a mais prevalente foi por câncer e mortes maternas. Dentre as mortes maternas, a causa mais prevalente foi síndrome hipertensiva. Identificamos a necessidade de reforçar urgentemente ações de promoção a saúde, prevenção de doenças e agravos, programas de rastreamento e diagnóstico precoce, garantindo serviços de qualidade e resolutivos em todos os níveis da atenção para todas as mulheres.
\end{abstract}

Palavras-chave: Mortalidade; Mortalidade materna; Mortalidade de mulheres em idade fértil. 


\begin{abstract}
The mortality of women of childbearing age and in the puerperal period corresponds to an important portion of female deaths in the country, with maternal mortality being an important indicator to assess the quality of health care offered to the population. Hypertension, hemorrhage, puerperal infections, diseases of the circulatory system complicated by pregnancy, childbirth and the puerperium and abortion are the main causes of maternal death in the country. Therefore, the objective of this work is to describe the deaths of women of childbearing age notified at the Barão de Lucena Hospital from January 2015 to October 2019. This is a descriptive, retrospective study of a quantitative character, carried out from death notification forms and hospital records of women of childbearing age, aged 10 to 49 years who died at Hospital Barão de Lucena. The study showed a higher number of deaths among women aged 40-49 years, of brown color, low education and without a steady partner. As for the causes of death, the most prevalent was cancer and maternal deaths. Among maternal deaths, the most prevalent cause was hypertensive syndrome. We identified the need to urgently reinforce health promotion actions, disease and disease prevention, screening programs and early diagnosis, ensuring quality and resolutive services at all levels of care for all women.
\end{abstract}

Keywords: Mortality; Maternal mortality; Mortality of women of childbearing age.

\title{
Resumen
}

La mortalidad de las mujeres en edad fértil y en el puerperio corresponde a una porción importante de las muertes de mujeres en el país, siendo la mortalidad materna un indicador importante para evaluar la calidad de la atención de salud que se brinda a la población. La hipertensión, la hemorragia, las infecciones puerperales, las enfermedades del sistema circulatorio complicadas por el embarazo, el parto y el puerperio y el aborto son las principales causas de muerte materna en el país. Por tanto, el objetivo de este trabajo es describir las defunciones de mujeres en edad fértil notificadas en el Hospital Barão de Lucena desde enero de 2015 hasta octubre de 2019. Se trata de un estudio descriptivo, retrospectivo, de carácter cuantitativo, realizado a partir de formularios de notificación de defunción y registros hospitalarios de mujeres en edad fértil de 10 a 49 años fallecidas en el Hospital Barão de Lucena. El estudio mostró un mayor número de muertes entre mujeres de 40 a 49 años, de color marrón, baja educación y sin pareja estable. En cuanto a las causas de muerte, las más prevalentes fueron el cáncer y las muertes maternas. Entre las muertes maternas, la causa más prevalente fue el síndrome hipertensivo. Identificamos la necesidad de reforzar urgentemente las acciones de promoción de la salud, enfermedades y prevención de enfermedades, programas de cribado y diagnóstico precoz, asegurando servicios de calidad y resolutivos en todos los niveles de atención para todas las mujeres.

Palabras clave: Mortalidade; Mortalidad maternal; Mortalidad de mujeres em edad fértil.

\section{Introdução}

No contexto mundial, a mulher ocupa um espaço valioso na sociedade moderna, sendo uma importante força de trabalho que move a economia, tendo o auge de suas atividades produtivas e reprodutiva, no período que chamamos de idade fértil. As mulheres em idade fértil correspondem ao grupo com faixa etária entre 10 e 49 anos de idade. Os óbitos de mulheres pertencentes a este grupo, equivalem a 16\% do total de óbitos femininos em todo Brasil (Pitilin \& Sbardelott, 2019).

Nos países em desenvolvimento esses óbitos são ainda mais numerosos, sinalizando que as mulheres que vivem nesses países estão mais vulneráveis a adoecimento e morte quando relacionado às mulheres, da mesma faixa etária, que vivem em países desenvolvidos (WHO, 2015). No Brasil, tais óbitos devem ser notificados ao Sistema de Informação de Mortalidade (SIM) em até 30 dias após a sua ocorrência, e a investigação de óbitos de Mulheres em Idade Fértil (MIF) é considerada oportuna, se for concluída e informada no SIM em até 120 dias após a sua ocorrência (Saúde Brasil, 2014).

Segundo dados da Pesquisa Saúde Brasil (2015), acidentes de transporte terrestre, homicídios, mortes maternas, suicídios e pneumonias foram as principais causas de morte de mulheres com idade entre 10 e 19 anos. Já em mulheres com idade entre 20 a 59 anos as principais foram doenças cerebrovasculares, câncer de mama, infarto agudo do miocárdio, diabetes mellitus e acidentes de transporte terrestre.

Em um estudo realizado na cidade de Guanambi/BA, foram encontradas diferenças entre o perfil da mortalidade de mulheres em idade fértil e a mortalidade materna. Enquanto nas primeiras os óbitos acontecem com maior frequência em mulheres com a faixa etária entre 40 a 49 anos, as mortes relacionadas ao período gravídico são mais comuns nas mulheres com idade entre 20 a 29 anos (Oliveira, Silva \& Teixeira, 2017). 
Mulheres morrem diariamente por complicações relacionadas ao período gravídico-puerperal (Gil e Gomes-sponholz, 2013), sendo a mortalidade materna um importante indicador para avaliar as condições de vida e assistência à saúde oferecidos à população. Assim, quanto menor a razão de mortalidade materna de uma região ou país, melhor a qualidade da atenção à saúde (Organização pan-americana da saúde, 2018).

Para Oliveira, Silva e Teixeira (2017), as mortes maternas não estão entre as dez primeiras causas de óbito entre mulheres com idade fértil, no entanto, a gravidade do problema é demonstrado quando se chama atenção para o fato de que a gravidez não é doença e que, na maioria das vezes, são consideradas evitáveis.

De acordo com o Manual dos Comitês de Mortes Maternas do Ministério da Saúde do Brasil (2009) a morte materna é o óbito da mulher durante a gestação ou até 42 dias após o término da gestação, independentemente da duração ou da localização da gravidez, sendo ocasionada por qualquer fator relacionado, agravado ou medidas tomadas em relação a ela.

Segundo a OMS a Razão de Morte Materna mundial diminuiu cerca de 44\% nos últimos 25 anos, saindo de um número de 385 para cada 100000 nascidos vivos em 1990 para 216 para cada 100.000 nascidos vivos em 2015. O Brasil não cumpriu o compromisso de chegar em 2015 com no máximo 35 óbitos maternos a cada 100 mil nascidos vivos (Organização pan-americana da saúde, 2018). Em 2018 a Razão de Morte Materna no Brasil foi de 59,1 óbitos para cada 100 mil nascidos vivos, sendo na região nordeste 67,1, número bem acima das metas firmadas com a ONU (Brasil, 2020).

A hipertensão, hemorragia, infecções puerperais, doenças do aparelho circulatório complicadas pela gravidez, parto e puerpério e o aborto são as cinco principais causas de morte materna no país (Ministério da saúde, 2009), sugerindo falhas no cuidado à saúde das mulheres, já que são consideradas mortes evitáveis e de possível resolução (Pitilin \& Sbardelott, 2019).

Entendendo o impacto sócio, econômico e demográfico da mortalidade em mulheres no período reprodutivo, torna-se importante conhecer as causas e as doenças relacionadas a esses óbitos notificados, com o intuito de contribuir para o conhecimento e auxiliar posteriores estudos que investiguem falhas no cuidado e contribua para melhorias permanentes na assistência à saúde. O estudo tem como objetivo descrever os óbitos de mulheres em idade fértil notificados no Hospital Barão de Lucena, em Recife-PE, no período de janeiro de 2015 à outubro de 2019.

\section{Metodologia}

Trata-se de um estudo retrospectivo de coorte documental com caráter quantitativo. Para Pereira, et al., (2018) no método quantitativo, a coleta de dados é realizada a partir de dados quantitativos ou numéricos e são analisados por meio de técnicas matemáticas como porcentagens, estatísticas e probabilidades.

O Estudo foi realizado a partir de fichas de notificação e investigação de óbitos e prontuários hospitalares de mulheres em idade fértil, de 10 a 49 anos, que foram a óbito no Hospital Barão de Lucena no período de janeiro de 2015 a outubro de 2019, em Recife-PE. A amostra contou com o universo total de óbitos notificados de mulheres em idade fértil ( $\mathrm{N}=222$ ), de acordo com os registros apurados na vigilância epidemiológica da instituição. A coleta de dados foi realizada no período de abril a setembro de 2020, onde foi solicitado ao setor de vigilância epidemiológica da instituição a ficha de notificação e investigação do óbito, assim como o número do prontuário de todas as mulheres com idade entre 10 e 49 anos que foram a óbito na instituição referida, no período do estudo. Em casos necessários, foi resgatado o prontuário hospitalar no setor de arquivos médicos para investigação das informações necessárias.

Os dados foram digitados e armazenados no Microsoft Office Excel, em computador próprio do pesquisador. Para análise descritiva, foram calculadas frequências simples e proporcionais. A relação entre variáveis foi analisada através do teste de qui-quadrado de Pearson e teste exato de Fischer, assumindo nível de significância para resultados com valor de $\mathrm{p}<0,05$. As análises estatísticas foram realizadas através do programa STATA versão 12. 
A pesquisa respeita a resolução 466/2012 do Conselho nacional de saúde, que tem com função implementar normas e diretrizes regulamentadoras de pesquisa envolvendo seres humanos. Tal pesquisa foi aprovada pelo Comitê de Ética em Pesquisa da Universidade Federal de Pernambuco localizado, com o CAAE n 29820620.0.0000.5208. A coleta de dados só foi iniciada após a submissão na Plataforma Brasil e aprovação do comitê de ética. Por se tratar de uma pesquisa de revisão de prontuários e fichas de notificação e investigação, foi dispensado o uso do Termo de Consentimento Livre e Esclarecido.

\section{Resultados e Discussão}

Com o intuito de descrever o perfil da mortalidade de mulheres em idade fértil no hospital Barão de Lucena em Recife-PE, esta pesquisa constatou que durante os anos analisados (janeiro de 2015 a outubro de 2019) foram registradas 222 mortes de mulheres em idade fértil, sendo o ano de 2017 com o número mais expressivo, 54 óbitos.

A Tabela 1 demonstra as características sociodemográficas da população presente na pesquisa. Em relação à faixa etária, notou-se um aumento no número de óbitos com o avançar da idade, estando à maioria dos episódios presente entre as mulheres de 40 a 49 anos, respondendo por 57.2\% dos casos, seguido pelas de 30 a 39 anos, com 25.2\%. Quanto à raça, mulheres de cor parda obtiveram o número mais expressivo, 75,5\%. Em relação a escolaridade, o maior número de óbitos ocorreu em mulheres que não possuíam escolaridade ou possuíam apenas o fundamental, 44,6\%. Quanto ao estado civil, observaram-se mais mortes nas mulheres sem parceiros fixos, que correspondem as solteiras, viúvas e divorciadas, com 51.8\%, seguida das com parceiro fixo, $27,3 \%$. E, do total de mulheres, $48.2 \%$ possuíam trabalho remunerado.

Tabela 1 - Perfil dos óbitos de mulheres em idade fértil segundo idade, raça, escolaridade, situação conjugal e ocupação no Hospital Barão de Lucena-Recife, 2015-2019.

\begin{tabular}{llll}
\hline Características & & $\mathbf{n}$ & $\mathbf{( \% )}$ \\
\hline \multirow{4}{*}{ Idade } & $10-14$ & 02 & 0.90 \\
& $15-19$ & 07 & 3.1 \\
& $20-29$ & 30 & 13.5 \\
& $30-39$ & 56 & 25.2 \\
Raça & $40-49$ & 127 & 57.2 \\
& Branca & 43 & 19.4 \\
& Parda & 161 & 72.5 \\
& Preta & 13 & 5.9 \\
Escolaridade & Ignorado & 05 & 2.2 \\
& fundamental/sem & 99 & 44.6 \\
& escolaridade & & \\
& Médio & 64 & 28.8 \\
Sit. Conjugal & Superior & 6 & 2.7 \\
& Ignorado & 53 & 23.9 \\
\hline \multirow{4}{*}{ Ocupação } & Parceiro fixo & 60 & 27.0 \\
& Sem parceiro fixo & 115 & 51.8 \\
& Ignorado & 47 & 21.2 \\
\hline & Trabalho não remunerado & 80 & 36.0 \\
& Trabalho remunerado & 107 & 48.2 \\
& Não trabalha & 05 & 2.2 \\
\hline
\end{tabular}

Fonte: Vigilância Epidemiológica do Hospital Barão de Lucena - Recife (2015 a 2019).

As características sociodemográficas encontradas no presente estudo se assemelham com outras pesquisas analisadas (Silva, et al., 2015; Oliveira, Rios \& Teixeira, 2017), onde ocorreu o aumento gradual do número de óbitos de acordo com a 
faixa etária, mortalidade mais frequente em mulheres que se autodeclararam pardas ou brancas, em mulheres sem parceiro fixo e com menor nível de escolaridade.

Segundo Silva, et al., (2015), A raça branca está associada a melhores condições de saúde e educação, obtendo mais acesso a saúde e maior tempo de escolaridade. Além de existir a relação da raça branca com melhores condições financeiras, sendo que os baixos níveis de renda aumentam os fatores de risco para morte.

Na Tabela 2, observa-se as principais causas que culminaram no óbito de mulheres em idade fértil, relatadas nas Declarações de Óbito. Relacionado a causa básica do óbito, o câncer foi a causa mais prevalente, com 65.8\%. As doenças crônicas e infecções ou doenças infeciosas possuíram, cada um, o percentual de 6.3 \% do total. Os óbitos sem definição, onde os corpos foram encaminhados para o Serviço de Verificação de Óbito (SVO) para definir a causa, correspondeu a 8.6\%. Quanto aos óbitos maternos, eles ocuparam o segundo lugar nas causas de óbito, obtendo o percentual de $14 \%$ da amostra.

Dentre os cânceres, os mais encontrados foram o de colo do útero/útero, correspondendo a 45 óbitos (31\% do total de cânceres) e câncer de mama ocupou o segundo lugar, com 44 casos. Tais dados podem ser justificados pelo fato de o estudo ter sido realizado em um hospital que é referência em ginecologia e fornecer à população tratamentos para doenças oncológicas.

Tabela 2 - Grupos de causas dos óbitos de mulheres de 10 a 49 anos.

\begin{tabular}{lcc}
\hline Causa do óbito & $\mathbf{n}$ & $(\%)$ \\
\hline Câncer & 146 & 66 \\
\hline $\begin{array}{l}\text { Infecção/doenças } \\
\text { infecciosas }\end{array}$ & 14 & 6,3 \\
\hline Doenças crônicas & 14 & \\
\hline óbito materno & 31 & 6,3 \\
\hline Sem definição & 10 & 4,5 \\
\hline Outros & 07 & 3,1 \\
\hline
\end{tabular}

Fonte: Setor de Vigilância Epidemiológica do Hospital Barão de Lucena - Recife-PE (2015 a 2019).

Estes resultados se assemelham a um estudo realizado no Piauí (2018), onde o câncer de mama e o câncer do colo do útero foram as neoplasias com maior incidência entre as mulheres estudadas. Estes dados demonstram comportamentos diferentes entre as taxas de mortalidade dessas duas neoplasias no Brasil, onde se observa aumento nas taxas de mortalidade por câncer de mama e diminuição da mortalidade para o câncer do colo do útero (Madeiro, et al., 2018).

Para Pereira e Somavilla (2020), um dos grandes problemas causadores da mortalidade da população de mulheres em idade fértil é o diagnóstico tardio e tratamento inadequado de doenças, como câncer e infecções sexualmente transmissíveis. Tal achado mostra falhas no serviço de saúde, principalmente no que diz respeito a prevenção, diagnóstico precoce e demora no tratamento adequado. 
O segundo grupo de situação clínica que mais levou a óbito mulheres em idade fértil foram as causas no período materno ou puerperal, como observado no Gráfico 1. Os óbitos maternos corresponderam a 31 óbitos do número total da pesquisa. Do número total, 11 mortes $(38,7 \%)$ não tiveram a causa de morte estabelecida, onde foi necessário enviar o corpo ao SVO para definir a causa da morte. Das mortes que obtiveram suas causas estabelecidas, síndromes hipertensivas foi a causa mais prevalente, correspondendo a $22,6 \%$ dos óbitos, esteatose hepática/pancreatite $9,7 \%$, causas infecciosas $6,5 \%$, hemorragia $6,5 \%$ e outras causas $16,1 \%$. As mortes ocorreram em grande parte dos casos durante o puerpério, em torno de $80,7 \%$, durante a gravidez $13 \%$ e aborto $6,4 \%$. Analisando-se o tipo de parto, observou-se uma frequência mais elevada da cirurgia cesariana, $74,1 \%$.

Gráfico 1. Causas básicas de Mortalidade materna.

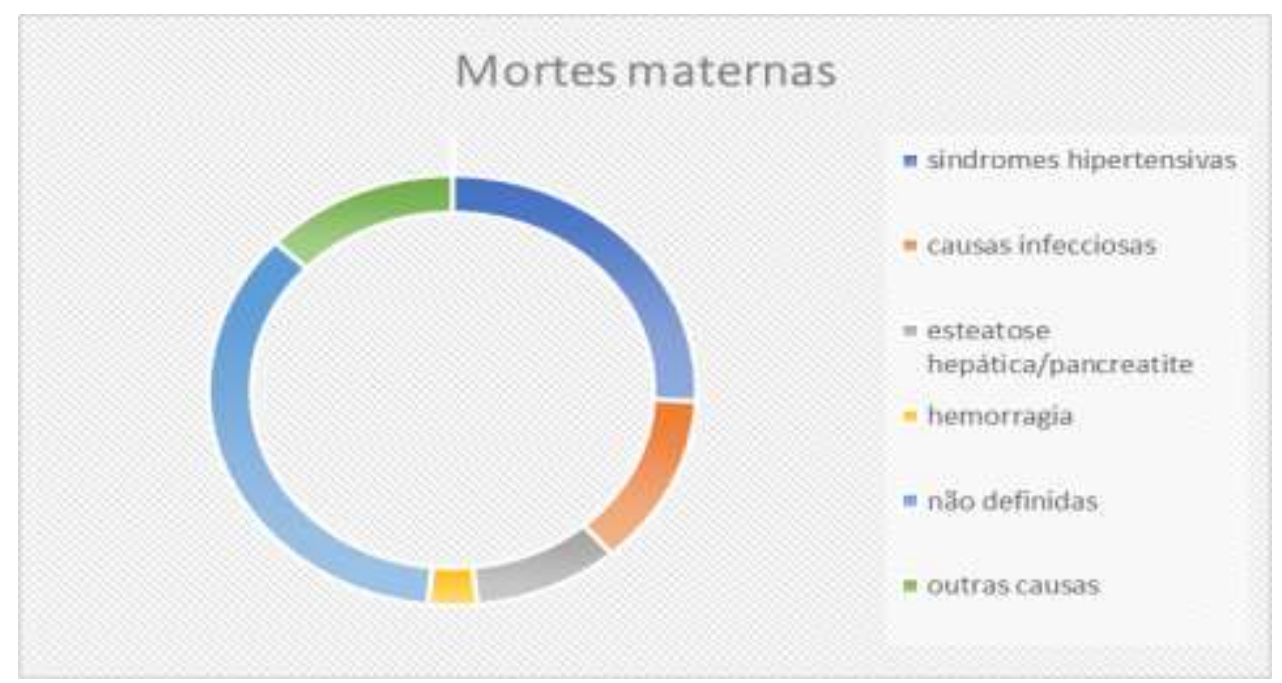

Fonte: Vigilância Epidemiológica do Hospital Barão de Lucena - Recife (2015 a 2019).

Em uma pesquisa realizada na cidade do Recife-PE entre os anos de 1994-2000, as causas mais frequentes de óbito foram hipertensão, hemorragia e infecção puerperal (Costa, et al., 2002). De acordo com o Boletim Epidemiológico (Brasil, 2020), as principais causas de óbitos maternos ocorridos no Brasil entre 1996-2018 por causas obstétricas diretas foram os mesmos, hipertensão, hemorragia e infecção puerperal.

Quanto ao tipo e momento do parto, os resultados corroboram com uma pesquisa realizada em outro hospital de alto risco da cidade do Recife, onde prevaleceram as mortes no período puerperal e partos cesáreos (Menezes, Bezerra e Bezerra, 2015). Devendo levar em consideração que se trataram de maternidades de alto risco, com maiores indicações para interrupção da gravidez por conta de fatores de risco para a mãe e o feto.

Ao investigarmos mortalidade materna dentre as variáveis sociodemográficas na amostra, encontramos maior proporção de óbitos maternos nas mulheres com faixa etária mais jovem de 10 a 29 anos, com significância estatística quando comparada às mais velhas, como visto na Tabela 3. O mesmo não ocorreu com as demais variáveis sociodemográficas. 
Tabela 3 - Comparação proporcional entre óbitos maternos e variáveis sociodemográficas na amostra estudada.

\begin{tabular}{lll}
\hline Variáveis & Óbito materno & Valor de P \\
\hline Idade & $19(61,3 \%)$ & $<0,001^{*}$ \\
$\mathbf{0 - 2 9}$ & $12(38,7 \%)$ & \\
$\mathbf{3 0 - 4 9}$ & & \\
& & $0,340^{* *}$ \\
Raça & $27(87,1 \%)$ & \\
Preta e parda & $04(12,9 \%)$ & \\
Branca & & $0,235^{* *}$ \\
\hline Escolaridade & & \\
Sem escolaridade/fundamental & $9(29 \%)$ & $0,247 * *$ \\
Ensino médio/superior & $11(35,5 \%)$ & \\
Ignorado & $11(35,5 \%)$ & \\
\hline Sit. Conjugal & & $0,568 * *$ \\
Tem parceiro & $03(10,4 \%)$ & \\
Não tem parceiro & $12(38 \%)$ & \\
Ignorado & $16(51,6 \%)$ & \\
\hline $\begin{array}{l}\text { Ocupação } \\
\text { Não trabalha } \\
\text { Trabalha }\end{array}$ & $0(0 \%)$ & \\
Ignorado & $20(64,5 \%)$ & \\
& $11(35,5 \%)$ & \\
\hline
\end{tabular}

*Teste qui-quadrado

** Teste exato de Fischer

Fonte: Setor de Vigilância Epidemiológica do Hospital Barão de Lucena - Recife-PE (2015 a 2019).

Para a variável relacionada à faixa etária, observamos que mulheres mais jovens pareceram apresentar maior chance de morte relacionada a causas maternas. Em relação as outras variáveis, houve predomínio da raça parda/preta, sem parceiro fixo, baixa escolaridade e que possuíam trabalho. Quanto a idade, o resultado se assemelha aos resultados encontrados em uma pesquisa realizada no Amazonas (Medeiros et al., 2018), onde os óbitos maternos foram mais frequentes em mulheres jovens.

Para Carvalho et al. (2016), O predomínio de mortalidade materna nas mulheres mais jovens deve ser interpretado levando-se em consideração que é nesse período que ocorrem a maioria das gestações. Já as mulheres mais velhas têm maior chance de serem portadoras de outras comorbidades, mais prevalentes com o passar dos anos (como o câncer, por exemplo).

Em pesquisa realizada no Mato Grosso, também apontou que a maior parte dos óbitos maternos ocorreram entre as mulheres pardas ou negras. Tal dado pode ser justificado por falha na assistência médica, considerada uma evidência de diferenças entre grupos étnico-raciais, tendo maior dificuldade e condições de acesso aos serviços de saúde de qualidade (Teixeira, et al., 2012).

A maior frequência de mulheres solteiras pode estar relacionada à desestruturação familiar, quebra de vínculo entre mãe e pai do bebê, bem como à falta de apoio das famílias (Medeiros, et al., 2018).

Outra questão importante é a quantidade de dados como ignorados, o que faz com que persista ainda problema de subnotificação de variáveis importantíssimas dentro do contexto social da saúde da mulher.

Neste contexto, observa-se a importância de ações que provoquem mudanças eficazes na assistência obstétrica, visto que boa parte destes óbitos poderiam ser evitados. A partir disto, é possível enxergar a mortalidade materna como um desafio à saúde pública que necessita de avanços e de melhorias na qualidade da assistência a essas mulheres (Scarton, et al., 2019). 


\section{Conclusão}

Conclui-se através deste estudo, que teve como objetivo analisar os óbitos de mulheres em idade fértil em um hospital terciário na cidade de Recife-PE no período de janeiro de 2015 a outubro de 2019, que a maioria dos óbitos de mulheres em idade fértil tinham entre 40-49 anos de idade, demonstrando aumento da mortalidade com o avançar da idade. Porém, quando analisamos os óbitos de origem materna, esta proporção é significantemente maior em mulheres mais jovens. Além disso, a maioria das mulheres eram da cor parda, não possuíam parceiro fixo, baixa escolaridade e possuíam trabalho remunerado.

A maioria das mortes analisadas ocorreram devido a câncer, principalmente ginecológico. Tal dado pode ser justificado por se tratar de um hospital referência em tratamentos ginecológicos, incluído oncológicos, sendo boa parte destes óbitos preveníeis ou passiveis de cura se diagnosticadas precocemente.

Vale ressaltar que na faixa etária analisada estão incluídas as mortes maternas, que é um importante indicador para analisar a qualidade da assistência à saúde, já que gravidez não é doença e, na maioria das vezes, são consideradas evitáveis se as mulheres tivessem acesso a profissionais e serviços qualificados para prevenir e tratar possíveis complicações.

Neste sentido, nota-se a necessidade de ações de promoção a saúde, prevenção de doenças e agravos, programas de rastreamento e diagnóstico precoce, garantindo serviços de qualidade e resolutivos em todos os níveis da atenção para todas as mulheres.

É importante destacar que informações não registradas nas declarações de óbito dificultou um pouco a análise dos dados, considerando que diminui a precisão dos dados. Neste sentido, se faz necessário reforçar aos profissionais responsáveis pelo preenchimento da Declaração de Óbito (DO), a importância do preenchimento correto e completo das DO e informações nos prontuários, a fim de melhorar a qualidade das as investigações de óbito, traçar o perfil epidemiológico e melhorar os serviços de notificação. Portanto, os achados indicam a urgência em aperfeiçoar a vigilância e atenção à saúde, além do desenvolvimento de estudos com desenhos robustos que avaliem os serviços e explicitem as iniquidades sociais e assistenciais entre diferentes grupos populacionais, expondo as condições de saúde das mulheres.

\section{Referências}

Brasil. Ministério da Saúde (2009). Manual dos comitês de mortalidade materna.

Brasil. Ministério da Saúde (2009). Guia de Vigilância Epidemiológica do Óbito Materno.

Brasil. Ministério da saúde (2015). Saúde Brasil 2014: Uma análise da situação de saúde e das causas externas.

Brasil. Ministério da saúde (2020). Boletim Epidemiológico 2020.

Carvalho, L. R. O., Fonseca, L. M. L., Coelho, M. T. C., de Souza Machado, M. G., de Carvalho, M. B., \& Vidal, C. E. L. (2016). Mortalidade de mulheres em idade fértil entre 1998 e 2012 na microrregião de Barbacena. Revista Interdisciplinar de Estudos Experimentais-Animais e Humanos Interdisciplinary Journal of Experimental Studies, 8(1).

Costa, A. A. R., Ribas, M. D. S. S. D. S., Amorim, M. M. R. D., \& Santos, L. C. (2002). Mortalidade materna na cidade do Recife. Revista Brasileira de Ginecologia e Obstetrícia, 24(7), 455-462.

Gil, M. M., \& Gomes-Sponholz, F. A. (2013). Declarações de óbitos de mulheres em idade fértil: busca por óbitos maternos. Revista Brasileira de Enfermagem, 66(3), 333-337.

Madeiro, A. P., \& Bilac, R. O. (2018). Mortalidade de mulheres em idade fértil no Piauí, Brasil, 2008-2012: causas básicas dos óbitos e fatores associados. CEP, 64001, 280 .

Medeiros, L. T., Sousa, A. M., Arinana, L. O., Inácio, A. S., Prata, M. D. L. C., \& Vasconcelos, M. N. G. (2018). Mortalidade materna no estado do Amazonas: estudo epidemiológico. Revista Baiana de Enfermagem, 32.

Menezes, M. L. N., Bezerra, J. O., \& Bezerra, J. F. O. Perfil epidemiológico dos óbitos maternos em hospital de referência para gestação de alto risco. Rev Rene. 2015; 15 (5): 714-21.

Oliveira, T. D. J., Rios, M. A., \& Teixeira, P. N. (2017). Mortalidade de mulheres em idade fértil na região de saúde de Guan ambi, BA. Mundo saúde (Impr.), $711-719$. 
Research, Society and Development, v. 10, n. 3, e30610313102, 2021

(CC BY 4.0) | ISSN 2525-3409 | DOI: http://dx.doi.org/10.33448/rsd-v10i3.13102

Organização Pan-americana da saúde (2018). Recomendações assistenciais para prevenção, diagnóstico e tratamento da hemorragia obstétrica.

Pereira, A. S. et al. (2018). Metodologia da pesquisa científica. UFSM

Pitilin, É. D. B., \& Sbardelotto, T. (2019). Mortalidade de Mulheres em Idade Reprodutiva: Estudo comparativo entre dois períodos. Rev. pesqui. cuid. fundam. (Online), 613-619.

Scarton, J., de Paula, S. F., de Andrade, G. B., Rangel, R. F., Ventura, J., \& de Siqueira, H. C. H. (2020). Maternal Mortality Profile: An Integrative Literature Review/Perfil da Mortalidade Materna: Uma Revisão Integrativa da Literatura. Revista de Pesquisa: Cuidado é Fundamental Online, 11(3), 816-822.

Silva, J. G., Moreira, K. F. A., Botelho, T. C. A., \& de Castro, T. M. (2015). Perfil da evitabilidade de óbitos de mulheres em idade fértil, de 2009 a 2013, em residentes de Porto Velho, Rondônia, Brasil. Revista Brasileira de Pesquisa em Saúde/Brazilian Journal of Health Research, 17(3), 49-59.

Pereira, M. K. K. A. (2020). Mortalidade Feminina-Perfil de Óbitos na Idade Fértil Não Associados à Maternidade. Revista de Epidemiologia e Controle de Infecção, $10(3)$

Teixeira, N. Z. F., Pereira, W. R., Barbosa, D. A., \& Vianna, L. A. C. (2012). Mortalidade materna e sua interface com a raça em Mato Grosso. Revista Brasileira de Saúde Materno Infantil, 12(1), 27-35.

World Health Organization (2015). Trends in Maternal Mortality: 1990 to 2015. Estimates developed by WHO, UNICEF, UNFPA, World Banka Group and The United Nations Population Division. 